\title{
Trial of Tramadol Plus Gabapentin for Opioid Detoxification
}

\author{
Hassan Ziaaddini ${ }^{1}$; Ahmad Ziaaddini ${ }^{2,}$; Neda Asghari ${ }^{1}$; Nozar Nakhaee $^{2}$; Mahin Eslami ${ }^{1}$ \\ ${ }^{1}$ Department of Psychiatry, Kerman University of Medical Sciences, Kerman, IR Iran \\ ${ }^{2}$ Kerman Neuroscience Research Center, Kerman University of Medical Sciences, Kerman, IR Iran \\ *Corresponding Author:Ahmad Ziaaddini, Kerman Neuroscience Research Center, Kerman University of Medical Sciences, P. O. Box: 7618668138, Kerman, IR Iran. Tel: +98-9133418244, E-mail: ziaaddini@me.com
}

Received: February 16, 2014; Revised: September 4, 2014; Accepted: October 26, 2014

\begin{abstract}
Background: Substance abuse or drug addiction is one of the most important health issues in every society, which can lead to physical and mental problems.

Objectives: This study aimed to compare the efficacy of tramadol plus gabapentin versus methadone use in the treatment of opiate withdrawal.

Patients and Methods: Consenting male subjects who fulfilled the DSM-4 criteria for opiate dependence syndrome (opium, residue, and heroin) were randomly assigned in two groups to receive tramadol plus gabapentin or methadone. Assessment tools were Adjective Rating Scale for Withdrawal (ARSW), Clinical Opiate Withdrawal Scale (COWS) and Visual Analogue craving Scale (VAS). Fifty-nine subjects were enrolled and evaluated on days 1,2,3, 4, 6, and 8 during their 10 days of admission. Twenty-nine participants received methadone and the other 30 received tramadol plus gabapentin for their treatment.

Results: Mean (SD) age of the patients in methadone group and tramadol plus gabapentin group were 33.9 (7.1) and 32.4. (8.1), respectively $(\mathrm{P}=0.462)$. The overall ARSW ( $\mathrm{P}$ value $=0.263)$ and COWS $(\mathrm{P}=0.862)$ scores between the two groups were comparable. The differences in the VAS score for craving between the two groups was marginally significant $(\mathrm{P}=0.057)$. The highest VAS score was at the third day of admission in both groups and it was generally higher in methadone group.

Conclusions: The severity of withdrawal syndrome in two groups was not significantly different. The craving was higher in the group receiving methadone from the second day of admission even though the usage amount was higher in the tramadol plus gabapentin group. The findings of this study suggest that the combination of tramadol plus gabapentin is an efficient method for opioid detoxification.
\end{abstract}

Keywords:Tramadol; Methadone; Randomized Controlled Trial; Humans; Opioid

\section{Background}

Substance abuse or drug addiction is one of the most important health issues in every society, which can lead to physical and mental problems (1). Detoxification is the first step after a patient is willing to go for the treatment and it has to be done in the most efficient and convenient way. The detoxification method has to overcome the withdrawal syndrome and help the patient to reduce the signs and symptoms as much as possible through the process $(2,3)$. Opioid withdrawal syndrome is a set of symptoms that occurs 6-8 hours after discontinuation of opioid class drugs. Different methods have been introduced for the treatment of opioid withdrawal syndrome, which include the opioid and non-opioid medications. They consist of opioid antagonists (naloxone, naltrexone); complete and partial opioid agonists (buprenorphine, methadone, levo$\alpha$-acetylmethadol), and $\alpha 2$ adrenergic agonists (clonidine, lofexidine) $(3,4)$. Also, their efficacy and success rate has been reported from $10 \%$ to $90 \%(5,6)$.

Tramadol is an atypical analgesic with a dual mechanism of action: serotonin and norepinephrine reuptake inhibition and modest $\mu$-opioid agonist. A prospective human laboratory study demonstrated that oral tra- madol doses of 200 or $400 \mathrm{mg}$ produced modest opioid withdrawal suppression among opioid-dependent research volunteers. These participants were maintained on subcutaneous morphine $(60 \mathrm{mg} / \mathrm{d}$ ) who experienced opioid withdrawal (3). Another drug is methadone, which is a synthetic narcotic analgesic compound developed in Germany just prior to World War II. After the war, methadone was studied and found to have effects similar to those of morphine but with longer duration (7). Different studies have shown that gabapentin is effective in the reduction of withdrawal syndrome $(8,9)$. Another study suggests that gabapentin reduces opioid use during a 10-day buprenorphine detoxification procedure (10). Rapid detoxification, which usually takes less than 5 days is more appropriate for patients using heroin (6). It is recommended that we avoid using anesthesia-assisted rapid opioid detoxification (AAROD) because of its mortality and morbidity in favor of evidence-based options for opioid dependence treatment (11). Transcutaneous electrical acupoint stimulation (TEAS) is an acceptable, inexpensive adjunctive treatment that is feasible to implement on an inpatient unit and may be a beneficial ad-

Copyright (C) 2015, Iranian Red Crescent Medical Journal. This is an open-access article distributed under the terms of the Creative Commons Attribution-NonCommercial 4.0 International License (http://creativecommons.org/licenses/by-nc/4.0/) which permits copy and redistribute the material just in noncommercial usages, provided the original work is properly cited. 
junct to pharmacological treatments for opioid detoxification (12). A retrospective cohort control study suggests that tramadol may be comparable to buprenorphine in the management of mild to moderately severe heroin withdrawal (13). Another study indicated a few clinical differences between parenteral buprenorphine and oral tramadol protocols when used in the management of acute heroin withdrawal. As a consequence, tramadol shows some promise as an opioid withdrawal management medication (14). However, in another research, tramadol was found to have limited detoxification efficacy in moderate to severe opioid withdrawal and substantial risk of seizures as compared to buprenorphine (15). In another study, tramadol compared to clonidine (in the management of heroin withdrawal) was more effective in preventing most withdrawal symptoms (16). A study that used the objective opioid withdrawal scale (OOWS) to compare the efficacy and safety of tramadol versus methadone showed that tramadol may be as effective as methadone in the control of withdrawal syndrome (17). Buprenorphine and methadone appear to be the most effective detoxification treatment compared to $\alpha 2$ adrenergic agonists (18).

\section{Objectives}

This study aimed to compare the efficacy of tramadol plus gabapentin versus methadone in treatment of the opiate withdrawal.

\section{Patients and Methods}

This clinical trial was conducted on male opiate dependent subjects admitted to Shahid Beheshti Hospital in Kerman (Iran) in 2013. Shahid Beheshti is a state-run psychiatric hospital with almost 200 beds. Inclusion criteria consisted of opioid dependency diagnosis based on DSMIV-TR, substance abuse for at least a year, and age range from 18 to 65 years. The eligible patients were enrolled consecutively. And the exclusion criteria were consumption of other medications such as BZD, TCA, corticosteroids, clonidine and codeine, iatrogenic dependency, critical medical conditions like diabetes, acute hepatitis, and other liver diseases. There were no subjects who met the exclusion criteria. Using census method, all eligible subjects were enrolled in the study. The study was approved by the Ethics Committee of Kerman University of Medical with the registration number of k90/530. Written informed consent was signed by all participants after a thorough explanation of the study by the physician. They were assured that their information would be kept confidential and they can leave the study whenever they want. A demographic questionnaire was then filled out at the first day of admission and the participants were randomly assigned (using random digit numbers) in two groups by a nurse. The physician was unaware of the patients group. The sample size was calculated to find out a $20 \%$ difference in withdrawal symptoms considering type I error of 0.05 and $80 \%$ power.

The control group received 10 to $30 \mathrm{mg}$ of methadone (syrup) in the first day and it was reduced 2.5 to $5 \mathrm{mg}$ each day from the second day. The other group received 200 to $300 \mathrm{mg}$ of tramadol (tablet) at the first and second day for patients using opium and residue and 300 to $400 \mathrm{mg}$ for patients using heroin divided in 3 doses, $100 \mathrm{mg}$ was added in the third day and was reduced to $50 \mathrm{mg}$ each day from the fourth day. Nine hundred miligram of gabapentin (capsule) was given to them each day divided in three doses. The patients were visited every day and the withdrawal syndrome and craving were assessed at the first, second, fourth, sixth, and eight day of admission using the COWS, ARSW and VAS (in Persian language), which their reliability were confirmed (19). The physician assessing was blind to the treatment group. However, the patient was aware of the treatment and all 59 subjects were enrolled in the study. Statistical analysis was done using the SPSS software (v19). The independent t test and chi-square were used to compare nominal and quantitative variables (Mann-Whitney U for non-parametric data). 2-way repeated measure analysis of variance (ANOVA) was used to compare the COWS, ARSW and VAS (which was converted to a number from 0 to 10). Because the data distribution was normal, we used square root transformation.

\section{Results}

Out of 59 subjects, 29 patients took the methadone treatment and 30 patients received the tramadol and gabapentin treatment protocol. As presented in Table 1, the mean age of the patients (33.9 years in methadone group and 32.4 years in tramadol and gabapentin group) and years of dependency ( 5.5 years in methadone group and 5.9 years in tramadol and gabapentin group) were comparable in both groups; however, the pre-intervention drug consumption was higher in tramadol and gabapentin treatment group $(\mathrm{P}=0.02)$. Marital status, degree of education and occupation were not significantly different in the two groups.

As demonstrated in Figure 1, the square of ARSW score has been compared between two treatment groups at the first, second, fourth, sixth, and eight day of admission. The differences in the ARSW score between two groups were not significant $(\mathrm{P}$ value $=0.263$ ). As demonstrated in Figure 2, the square of COWS score has been compared between two treatment groups at the first, second, fourth, sixth, and eight day of admission. The differences in the COWS score between two groups were not significant ( $P$ value $=0.862)$. As demonstrated in Figure 3, the square of VAS score has been compared between two treatment groups at the first, second, fourth, sixth, and eight day of admission. The differences in the VAS score between two groups were nearly significant $(P=0.057)$. In methadone group an upsurge of clinical signs was seen in the third day of treatment, whilst in tramadol and gabapentin treatment group a smooth decrease in severity of clinical signs was seen from the onset of treatment. 
Ziaaddini H et al.

Table 1. The Mean Age of the Patients and Years of Dependency Were Comparable in Both Groups

\begin{tabular}{|c|c|c|c|}
\hline Characteristics & Methadone & Tramadol + Gabapentin & P Value \\
\hline Age, $y^{a}$ & $33.9 \pm 7.1$ & $32.4 \pm 8.1$ & 0.462 \\
\hline Years of dependency ${ }^{a}$ & $5.5 \pm 6.2$ & $5.9 \pm 4.8$ & 0.538 \\
\hline Usage amount (converted to methadone), $\mathrm{mg} / \mathrm{d}^{\mathrm{a}}$ & $99.1 \pm 112$ & $163.2 \pm 147$ & 0.02 \\
\hline Marital status & & & 0.937 \\
\hline Single & 8 & 8 & \\
\hline Married & 21 & 22 & \\
\hline Type of drug & & & 0.003 \\
\hline Opium & 21 & 18 & \\
\hline Residue & 8 & 3 & \\
\hline Heroin & 0 & 9 & \\
\hline Degree of Education & & & 0.165 \\
\hline None & 1 & 5 & \\
\hline Some education & 17 & 10 & \\
\hline High school diploma & 9 & 12 & \\
\hline University degree & 2 & 3 & \\
\hline Occupation & & & 0.155 \\
\hline Unemployed & 0 & 3 & \\
\hline Part-time & 25 & 21 & \\
\hline Full-time & 4 & 6 & \\
\hline
\end{tabular}

a Data are presented as Mean \pm SD.

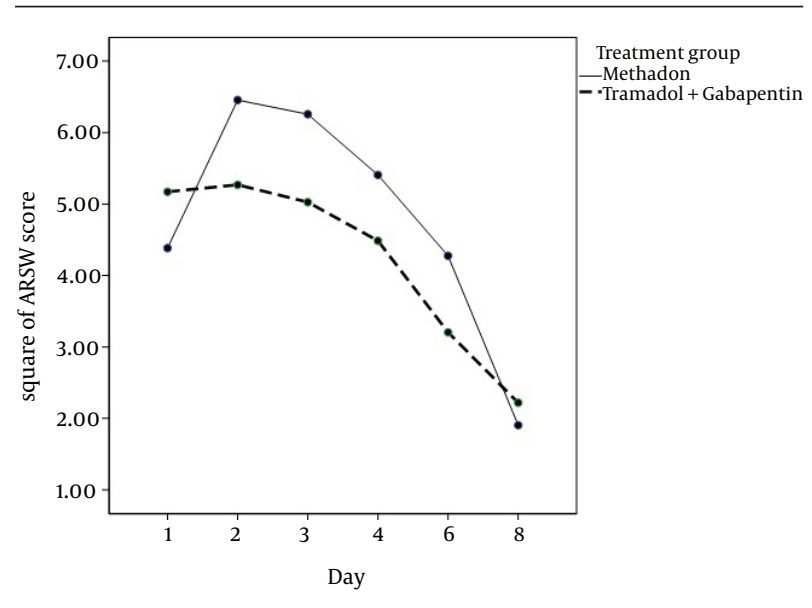

Figure 1. The Square of Adjective Rating Scale for Withdrawal Score has Been Compared in the Two Treatment Groups at the First, Second, Fourth, Sixth, and Eight Day of Admission

\section{Discussion}

This clinical trial was conducted on 59 male opiate dependent subjects, which were treated in two different groups. Twenty-nine subjects received methadone and 30 subjects received tramadol and gabapentin for treatment. All the patients were aware of their medications. Although the usage amount (which was much higher in the tramad-

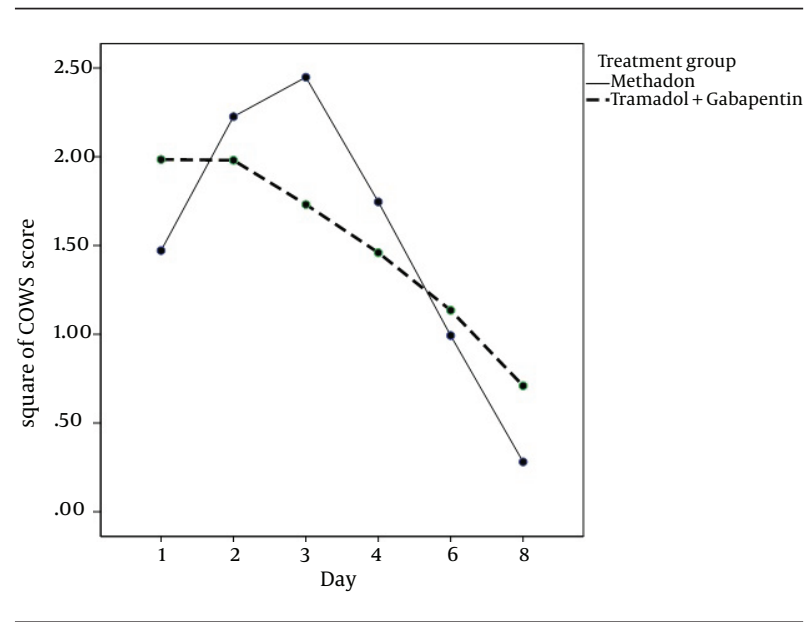

Figure 2. The Square of Clinical Opiate Withdrawal Scale Score has Been Compared in the Two Treatment Groups at the First, Second, Fourth, Sixth, and Eight Day of Admission

ol plus gabapentin group, Pvalue =0.02) and type of drug abused $(P$ value $=0.003$ ) was significantly different in the two groups but the differences in the overall ARSW (P value $=0.263)$ and COWS $($ P value $=0.862)$ scores between the groups were not significant. The mean square of ARSW and COWS score at days 1 to 8 were similar. 


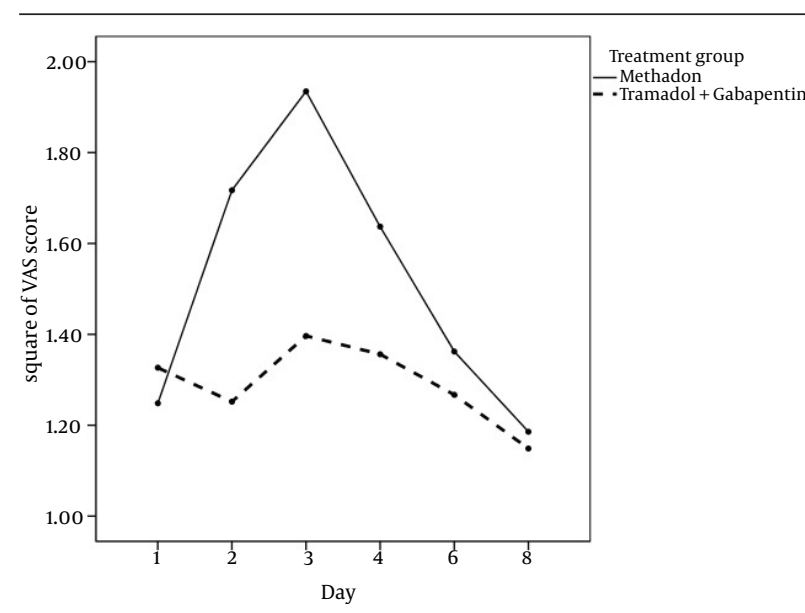

Figure 3. The Square of Visual Analogue Craving Scale Score has Been Compared in the Two Treatment Groups at the First, Second, Fourth, Sixth, and Eight Day of Admission

These data suggest that the severity of withdrawal syndrome in the two groups was not significantly different. A similar study, which used the OoWS instrument for assessment of withdrawal syndrome to compare the efficacy and safety of tramadol versus methadone for treatment of opiate withdrawal showed that tramadol may be as effective as methadone in the control of withdrawal syndrome (17). The differences in the VAS score between two the groups were near significant $(P$ value $=0.057$ ) And the craving is higher in the group receiving methadone from the second day of admission even though the usage amount was higher in the tramadol and gabapentin group. The highest craving was at the third day of admission in the methadone group (Figure 3). In this clinical trial, we added gabapentin to tramadol to reduce the risk of seizure by tramadol and to reduce the withdrawal syndrome intensity as been noticed in different studies $(8,9)$. Another study suggests that gabapentin reduces opioid use during a 10-day buprenorphine detoxification procedure (10). The tramadol plus gabapentin protocol used reduced craving compared to methadone in our study. Most of the studies that compared tramadol with buprenorphine suggested that tramadol may be as effective as buprenorphine in the management of opioid withdrawal syndrome (13-15). In another study, tramadol has been compared to clonidine in the management of heroin withdrawal and tramadol was more effective in preventing most withdrawal symptoms (16).

The findings of our study suggest that the combination of tramadol with gabapentin is an efficient method for opioid detoxification. The strong point of the study is the use of 3 different assessments (ARSW, COWS, and VAS) in the two groups. The weak point of the study is the significant differences in respect to the types and amount of drug abuse in two groups. It is recommended for further studies to assess the patient's long-term substance use after the detoxification period. The limitation of the study was that the two groups had significant differences in respect to the types and amount of drug abuse and the variables were not adjusted in the final analysis.

\section{Acknowledgements}

The authors wish to thank the staff of Kerman University of Medical Sciences and Shahid Beheshti Hospital for their support.

\section{Funding/Support}

This research was financially supported by Kerman University of Medical Sciences, Kerman, Iran.

\section{References}

1. Ziaaddini H, Sharifi A, Nakhaee N, Ziaaddini A. The Prevalence of at Least One-Time Substance Abuse among Kerman Pre-university Male Students. Addict Health. 2010;2(3-4):103-10.

2. Gowing L, Ali R, White J. Buprenorphine for the management of opioid withdrawal. Cochrane Database Syst Rev. 2006(2):CD002025.

3. Sadock BJ, Sadock VA, Ruiz P. Kaplan and Sadock's Comprehensive Textbook of Psychiatry. 9 edphiladelphia: Lippincott Williams \& Wilkins; 2009.

4. Ries RK, Fiellin DA, Miller SC, Saitz R. Principles of Addiction Medicine. 4 edPhiladelphia: Lippincott Williams \& Wilkins; 2009.

5. Mokri A. Brief overview of the status of drug abuse in Iran. Arch Iranian Med. 2002;5(3):184-90.

6. Salehi M, Amanatkar M, Barekatain M. Tramadol versus methadone for the management of acute opioid withdrawal: an addon study. J Rese Med Sci. 2006;11(3):185-9.

7. Lowinson JH, Ruiz P, Millman RB, Langrod JG. Substance Abuse A comprehensive Textbook.Philadelphia: Lippincott Williams \& Wikins; 2005.

8. Mobasher M, Ziaaddini H, Sabzvari F, Sadeghipour S. The Effect of Gabapentin on Withdrawal Syndrome, Psychiatric Disorders and Electroencephalogram of Opium Addicts during the Detoxification Period. Iran J Pharm Res. 2010;4:215-23.

9. Ziaaddini H, Qahestani A, Moin Vaziri M. Comparing Symptoms of Withdrawal, Rapid Detoxi-fication and Detoxification with Clonidine in Drug Dependent Patients. Addict Health. 2009;1(2):63-8.

10. Sanders NC, Mancino MJ, Gentry WB, Guise JB, Bickel WK Thostenson J, et al. Randomized, placebo-controlled pilot trial of gabapentin during an outpatient, buprenorphineassisted detoxification procedure. Exp Clin Psychopharmacol. 2013;21(4):294-302.

11. Centers for Disease C, Prevention.. Deaths and severe adverse events associated with anesthesia-assisted rapid opioid detoxification--New York City, 2012. MMWR Morb Mortal Wkly Rep. 2013;62(38):777-80.

12. Meade CS, Lukas SE, McDonald LJ, Fitzmaurice GM, Eldridge JA, Merrill N, et al. A randomized trial of transcutaneous electric acupoint stimulation as adjunctive treatment for opioid detoxification. J Subst Abuse Treat. 2010;38(1):12-21.

13. Tamaskar R, Parran TV, Jr., Heggi A, Brateanu A, Rabb M, Yu J. Tramadol versus buprenorphine for the treatment of opiate withdrawal: a retrospective cohort control study. J Addict Dis. 2003;22(4):5-12.

14. Threlkeld M, Parran TV, Adelman CA, Grey SF, Yu J. Tramadol versus buprenorphine for the management of acute heroin withdrawal: a retrospective matched cohort controlled study. Am J Addict. 2006;15(2):186-91.

15. Chawla JM, Pal H, Lal R, Jain R, Schooler N, Balhara YP. Comparison of efficacy between buprenorphine and tramadol in the detoxification of opioid (heroin)-dependent subjects. J Opioid Manag. 2013;9(1):35-41.

16. Chattopadhyay S, Singh OP, Bhattacharyya A, Sen S, Roy P, Deb- 


\section{Ziaaddini $\mathrm{H}$ et al.}

nath $\mathrm{S}$. Tramadol versus clonidine in management of heroin withdrawl. Asian J Psychiatr. 2010;3(4):237-9.

17. Zarghami M, Masoum B, Shiran MR. Tramadol versus methadone for treatment of opiate withdrawal: a double-blind, randomized, clinical trial. J Addict Dis. 2012;31(2):112-7.

18. Meader N. A comparison of methadone, buprenorphine and alpha(2) adrenergic agonists for opioid detoxification: a mixed treatment comparison meta-analysis. Drug Alcohol Depend. 2010;108(1-2):110-4

19. Ziaaddini H, Nasirian M, Nakhaee N. Comparison of the efficacy of buprenorphine and clonidine in detoxification of opioiddependents. Addict Health. 2012;4(3-4):79-86. 